\title{
M1 properties of the few-nucleon systems
}

\section{Rimantas Lazauskas*}

IPHC, IN2P3-CNRS/Université Louis Pasteur BP 28, F-67037 Strasbourg Cedex 2, France

E-mail: rimantas.lazauskas@ires.in2p3.fr

\section{Young-Ho Song}

Department of Physics, Duke University, Durham, NC 27708, USA

E-mail: yhsong@phy.duke.edu

\section{Tae-Sun Park}

Department of Physics and BAERI, Sungkyunkwan University, Suwon 440-746, Korea

E-mail: tspark@kias.re.kr

\begin{abstract}
Magnetic dipole (M1) properties of the $A=2-4$ nucleon systems is studied for realistic nuclear Hamiltonians and meson exchange currents derived up to $\mathrm{N}^{3} \mathrm{LO}$ using heavy baryon chiral perturbation theory. In particular, for the first time thermal neutron capture on ${ }^{3} \mathrm{He}$ calculations have been performed using fully rigorous nuclear wave functions.
\end{abstract}

6th International Workshop on Chiral Dynamics, CD09

July 6-10, 2009

Bern, Switzerland

\footnotetext{
*Speaker.
} 


\section{Introduction}

Magnetic dipole (M1) properties of few-nucleon system has been extensively investigated during the last two decades. The interest in low energy M1 transitions is additionally stimulated by the fact that in some cases, due to the internal symmetry of the nuclear system, single-nucleon (1B) currents can be strongly suppressed. Then meson exchange currents, closely related with nucleon-nucleon interaction, become visible enabling to test our understanding of the mechanism underlaying the nuclear interactions with electro-weak probes.

Description of such systems using standard nuclear physics approach (SNPA) based on mesonexchange currents and high-precision phenomenological potentials is unambiguous, due to the failure to establish an unique meson-exchange current associated with phenomenological part of the nuclear potential at short distances. However such systems presents an ideal laboratory to test effective field theories (EFTs), and in this regard, M1 properties have been extensively studied $[1,2,3,4,5,6,7]$. One of the examples of huge success of EFT is the ability to describe $\sigma_{n p}$, the capture cross section of the $n p \rightarrow d \gamma$ process, at threshold with $1 \%$ accuracy by applying heavy-baryon chiral perturbation theory (HBChPT) up to next-next-next-to the leading order or $\mathrm{N}^{3} \mathrm{LO}$ [1]. In this work, we have extend our up-to $\mathrm{N}^{3} \mathrm{LO}$ HBChPT description of the M1 properties to $A=3-4$ domain. By taking the magnetic moments of ${ }^{3} \mathrm{H}$ and ${ }^{3} \mathrm{He}$ as an input to fix the coefficients of the contact-term operators, a completely parameter-free theory predictions will be made for the total cross section $\sigma_{n d}$ and the photon circular polarization parameter $R_{c}$ of the so called den process (the thermal neutron capture on deuterons $n d \rightarrow{ }^{3} \mathrm{H} \gamma$ ) as well as for the total cross section $\sigma_{n^{3} \mathrm{He}}$ of the hen process (the thermal neutron capture on ${ }^{3} \mathrm{He}$ nuclei $n^{3} \mathrm{He} \rightarrow{ }^{4} \mathrm{He} \gamma$ ). In this framework we will also revisit the theory predictions for the two-body observables: deuteron magnetic moment $\mu_{d}$ and $\sigma_{n p}$.

\section{Current operator}

We are interested in magnetic dipole operator

$$
\mu_{1}(q)=(i q / \sqrt{6 \pi})^{-1} \hat{T}_{10}^{M a g}(q)
$$

where $\hat{T}_{10}^{M a g}(q)$ is defined in [8], $q^{\mu}=(\omega, \mathbf{q})$ is the momentum carried out by the photon, and $q \equiv|\mathbf{q}|$. This operator give rise to magnetic moments ( $q^{\mu}=0$ in this case) of the nuclei but also is largely dominant contributor for the thermal neutron capture on ${ }^{1} \mathrm{H},{ }^{2} \mathrm{H}$ and ${ }^{3} \mathrm{He}$ nuclei, since at these energies both initial and final state have positive parities, while angular momenta of available initial and final states satisfy $\left|J_{i}-J_{f}\right| \leq 1$.

We derive the M1 operator in HBChPT by considering only the nucleons and pions as pertinent degrees of freedom, while integrating all the other massive fields out. In HBChPT the electromagnetic currents and M1 operator are expanded systematically with increasing powers of $Q / \Lambda_{\chi}$, where $Q$ stands for the typical momentum scale of the process and/or the pion mass, and $\Lambda_{\chi} \sim 4 \pi f_{\pi} \sim m \sim 1 \mathrm{GeV}$ is the chiral scale, $f_{\pi} \approx 92.4 \mathrm{MeV}$ is the pion decay constant, and $m$ is the nucleon mass. We remark that, while the nucleon momentum $p_{i}$ is of the order of $Q$, its kinetic energy $\left(T=p_{i}^{2} / m\right)$ is of order of $Q^{2} / m$, and consequently the four-momentum of the emitted photon $q^{\mu}=(\omega, \mathbf{q})$ should also be counted as $\mathscr{O}\left(Q^{2} / m\right)$. In this work we include all the contributions 
up to $\mathrm{N}^{3} \mathrm{LO}$, where $\mathrm{N}^{v} \mathrm{LO}$ denotes terms of order of $\left(Q / \Lambda_{\chi}\right)^{v}$ compared to the leading one-body contribution. It is worth mentioning that there exist a different power counting scheme where the nucleon mass is regarded as heavier than the chiral scale, see refs. [9] for the details. However, the use of this alternative counting scheme would not affect the results to be reported in this article since the difference between the two counting schemes would appear only at orders higher than explicitly considered here $\left(\mathrm{N}^{3} \mathrm{LO}\right)$. Full expressions for the current operators we use have been provided explicitly in our previous study [7, 10], thus we refer an interested reader to these works.

\section{Wave functions}

In order to calculate transition matrix elements it is imperative to dispose of accurate wave functions both for the elastic scattering states (which correspond the initial state in radiative capture calculations) as well as for the bound states (which are compulsory both for capture and magnetic moment calculations). Wave functions we use are the mathematically rigorous solutions of underlaying non-relativistic Quantum mechanics problem for realistic nuclear Hamiltonians. They are obtained by solving corresponding few-body problem using Faddeev (for 3-body case) [11] and Faddeev-Yakubovski (4-body case) [12] equations in configuration space. Here we shortly present 4-nucleon Faddeev-Yakubovski (FY) equations, which are formulated in isospin formalism, i.e. considering proton and neutron as two isospin degenerate states of the same particle, nucleon with the mass, which was fixed to $\hbar^{2} / m=41.47 \mathrm{MeV} \cdot \mathrm{fm}^{2}$. FY equations have been rewritten in form suitable to incorporate long-ranged Coulomb force as well as three-nucleon forces. With the threebody force $V_{123}=V_{12}^{3}+V_{23}^{1}+V_{31}^{2}$, the FY equations for Faddeev-Yakubovski amplitudes (FYA) $K \equiv K_{12,3}^{4}$ and $H \equiv H_{12}^{34} \operatorname{read}[13]$

$$
\begin{aligned}
& \left(E-H_{0}-V_{12}-\sum_{i<j} V_{i j}^{C}\right) K=V_{12}\left(P^{+}+P^{-}\right)[(1+Q) K+H]+\frac{1}{2}\left(V_{23}^{1}+V_{31}^{2}\right) \Psi, \\
& \left(E-H_{0}-V_{12}-\sum_{i<j} V_{i j}^{C}\right) H=V_{12} \tilde{P}[(1+Q) K+H],
\end{aligned}
$$

where $V_{i j}$ and $V_{i j}^{C}$ are, respectively, the short-ranged part and the Coulomb-dominated long-range part of the interaction between the $i$-th and $j$-th nucleons. $P^{+}=\left(P^{-}\right)^{-1} \equiv P_{23} P_{12}, Q \equiv-P_{34}$ and $\tilde{P} \equiv P_{13} P_{24}=P_{24} P_{13}$, where $P_{i j}$ is the particle permutation operator. In terms of the FYAs, the total wave function of an $A=4$ system is given by

$$
\Psi=\left[1+\left(1+P^{+}+P^{-}\right) Q\right]\left(1+P^{+}+P^{-}\right) K+\left(1+P^{+}+P^{-}\right)(1+\tilde{P}) H .
$$

We expand $K$ and $H$ in terms of the tripolar harmonics $Y_{i}^{\alpha}\left(\hat{x}_{i}, \hat{y}_{i}, \hat{z}_{i}\right)$, which comprise spins and isospins of the nucleons as well as angular momentum variables,

$$
\Phi_{i}\left(\vec{x}_{i}, \vec{y}_{i}, \vec{z}_{i}\right)=\sum_{\alpha} \frac{\mathscr{F}_{i}^{\alpha}\left(x_{i}, y_{i}, z_{i}\right)}{x_{i} y_{i} z_{i}} Y_{i}^{\alpha}\left(\hat{x}_{i}, \hat{y}_{i}, \hat{z}_{i}\right),
$$

where $\Phi$ stands for either $K$ or $H$, and the subscript $i$ denotes the particle-grouping class (among the four nucleons).

The partial components $\mathscr{K}_{i}^{L S T}$ and $\mathscr{H}_{i}{ }^{L S T}$ are expanded in the basis of three-dimensional splines. One thus converts integro-differential equations into a system of linear equations. More detailed discussion on the numerical method and equations used can be found in [13]. 


\section{Results}

Our EM current operator up to the order $\mathrm{N}^{3} \mathrm{LO}$ involves two irreducible contact terms, which should mimic effects due to the massive fields (or short range), and thus contains two low-energy constants (LECs), denoted by $g_{4 s}$ and $g_{4 v}$. The values of these LECs can be fixed rigorously only by solving QCD at low-energy, which is not feasible at the moment. Therefore we choose more phenomenological option to determine LECs by requiring to reproduce ${ }^{3} \mathrm{H}$ and ${ }^{3} \mathrm{He}$ magnetic dipole moments ${ }^{1}$. In this paper we present results based on nuclear wave functions obtained for five different Hamiltonians. These Hamiltonians are: Argonne potential AV18 [14], the chiral $\mathrm{N}^{3} \mathrm{LO}$ potential of Idaho group I-N3LO [15], the semi-realistic non-local configuration space potential INOY derived by Doleschall [16]. Urbana type [17] three nucleon force have been also included in calculations with AV18 and I-N3LO potentials. For AV18 potential standard parameters of UIX parametrization have been used, while for the case of I-N3LO+UIX* we have slightly changed the parameter $A_{2 \pi}=-0.03827 \mathrm{MeV}$ of the original UIX parametrization. The last adjustment have been done in order to to reproduce the triton binding energy.

Table 1: Predictions for the deuteron $\left({ }^{2} \mathrm{H}\right)$ magnetic moment (in nuclear magnetons), the capture cross sections for the thermal neutron capture on protons, deuterons and ${ }^{3} \mathrm{He}$ as well as photon circular polarization parameter for the neutron capture on deuteron $\left(R_{c}\right)$. These calculations have been realized by fixing contact terms of the meson exchange current (MEC) in order to reproduce magnetic moments of the triton $\left({ }^{3} \mathrm{H}\right)$ and ${ }^{3} \mathrm{He}$. For two and three nucleon systems these values turns to be insensitive to the cut-off parameter in the interval $\Lambda=(500,900) \mathrm{MeV}$; if however variation was larger than one affecting the last significant digit its value is provided in parentheses. For $\sigma_{n^{3}} \mathrm{He}$ capture variation is large and is given as \pm value.

\begin{tabular}{cccccc} 
Model & $\mu\left({ }^{2} \mathrm{H}\right)$ & $\sigma_{n p}(m b)$ & $\sigma_{n^{2} \mathrm{H}}(m b)$ & $R_{c}$ & $\sigma_{n^{3} \mathrm{He}}(\mu b)$ \\
\hline AV18 & 0.8575 & $331.9(1)$ & $0.680(3)$ & -0.435 & $80.0 \pm 12.2$ \\
AV18+UIX & 0.8604 & $330.6(2)$ & $0.478(3)$ & -0.458 & $57.3 \pm 7.9$ \\
INOY & 0.8585 & $330.6(2)$ & $0.498(3)$ & -0.465 & $34.3 \pm 4.5$ \\
I-N3LO & 0.8574 & $330.4(3)$ & $0.626(2)$ & -0.441 & $49.4 \pm 8.5$ \\
I-N3LO+UIX* & $0.8590(1)$ & $329.7(3)$ & $0.477(3)$ & $-0.468(1)$ & $44.4 \pm 6.7$ \\
\hline Exp.: & 0.8574 & $332.6 \pm 0.7[19]$ & $0.508 \pm 0.015[20]$ & $-0.420 \pm 0.030[21]$ & $55 \pm 3[22]$ \\
& & & & & $54 \pm 6[23]$
\end{tabular}

The first feature to stress is the independence of the obtained results to the cut-off parameter $\Lambda$, which have been varied in the interval $(500,900) \mathrm{MeV}$. Variation due to the cut-off is of the order of numerical accuracy for two and three nucleon M1 observables. This gives a sound support for the HBChPT currents and the power counting scheme in use. Situation is worse for the hen cross section. Here cut-off dependence is quite important. This drawback, as discussed [18], is due to the complexity of this system. The major components of the initial $n+{ }^{3} \mathrm{He}$ scattering state and final ${ }^{4} \mathrm{He}$ bound state wave functions belong to the [31] and the [4] spatial permutation symmetry groups

\footnotetext{
${ }^{1}$ One should notice that other experimental data can be used to fix LECs. One practical option is to choose ${ }^{2} \mathrm{H}$ magnetic moment and $n p$ thermal capture cross section as reference, enabling to fix the two LECs independently. However very accurate $n p$ cross section measurement is unfeasible. Therefore selection of the ${ }^{3} \mathrm{H}$ and ${ }^{3} \mathrm{He}$ magnetic moments, which are known with 9-digit accuracy, to determine LECs has advantage enabling to test our results without performing error propagation procedure.
} 
respectively, they cannot be connected through the $r$-independent operators. Therefore leading 1body current operator is strongly suppressed, partial suppression also appears in the leading 2-body one-pion exchange contributions. Moreover there is a strong cancelation between single nucleon and meson-exchange currents, which permits to blow up importance of the higher order terms in the power counting scheme.

Second observation is strong model dependence of the calculated capture observables. There is more than factor 2 difference between calculated $n^{3} \mathrm{He} \rightarrow{ }^{4} \mathrm{He} \gamma$ cross section for AV18 and INOY models. These discrepancies are due to the mismatches from the long-range contributions. Long-range parts of the initial and final state wave functions are governed by the relevant effective range parameters (ERPs) as binding energies (for the bound states) and scattering lengths (for the scattering states). Not all the Hamiltonians employed are able to reproduce correctly experimental values of ERPs for A=3-4 systems. Nevertheless the low energy ERPs can be related, it is well observed correlation of the singlet $n$ - $d$ scattering length with trinucleon binding energy (Philips line), as well as correlation between binding energies of the alpha-particle and ${ }^{3} \mathrm{H}$ nucleus (Tjon line). Correlation have been also observed between thermal neutron-deuteron capture cross section and ${ }^{3} \mathrm{H}$ binding energy by Friar et al. [24], this correlation for our results is demonstrated in left pane of the Fig. 1. The $n^{3} \mathrm{He} \rightarrow{ }^{4} \mathrm{He} \gamma$ reaction is a complicated four-body process that may involve a large number of ERPs. Still we found that $\sigma_{n^{3}} \mathrm{He}$ is almost proportional to the quantity

$$
\zeta \equiv\left[q\left(a_{n \mathrm{He} 3} / r_{\mathrm{He} 4}\right)^{2}\right]^{-2.75}
$$

where $q=\mathrm{BE}\left({ }^{4} \mathrm{He}\right)-\mathrm{BE}\left({ }^{3} \mathrm{He}\right)$ is the $Q$ value, $a_{n \mathrm{He} 3}$ is the real part of the spin-triplet $n-{ }^{3} \mathrm{He}$ scattering length and $r_{\mathrm{He} 4}$ is the point-proton rms radius of ${ }^{4} \mathrm{He}$. This proportionality is demonstrated in the right pane of Fig. 1. Considering these correlations one can establish model-independent predictions, which correspond to the experimental ERPs. We deduce $\sigma_{n d}=0.490 \pm 0.008 \mathrm{mb}$ and $R_{c}=-0.462 \pm 0.03$ for thermal $n^{2} \mathrm{H} \rightarrow{ }^{3} \mathrm{H} \gamma$ and $\sigma_{n^{3}} \mathrm{He}=54.9 \pm 9.1 \mu b$, which are in reasonable agreement with experimentally measured values.

As seen in the table determined values for two nucleon M1-properties are also in reasonable agreement with experimental data, however for $n p$ capture cross section they are up to $3 \sigma$ below experimental point. Moreover visibly agreement worsens as one includes three nucleon interaction, when calculating trinucleon wave functions. This feature suggest that probably three-nucleon currents, which enter in our power counting at $\mathrm{N}^{4} \mathrm{LO}$, turn to be the most important omitted contribution. This issue should be solved in our future works.

Summarizing one should stress that our hybrid approach provides results, which turns to be in a rather good agreement with experimental data. The small discrepancies found for M1 properties of two and three nucleon systems are of the similar magnitude as provided by the best standard approach calculation [25]. Our results turns to be less cut-off dependent and closer to the experimental values than ones presented in the recent study by Pastore et al. [26], which is based on a similar approach we use, however which in addition explicitly includes $\Delta$-isobar degrees of freedom. One should stress however that calculations performed by Sadeghi [5] using pionless theory turns to be in perfect agreement with experiment for den process. This feature is very surprising, as the last approach turns to be the simplest and roughest Effective Field theory application. This issue should be carefully studied by testing M1 properties beyond low energy radiative capture pro- 
cess. For example pionless approach used in [5] should provide deuteron with magnetic moment $\mu_{d}=\mu_{p}+\mu_{n}=0.87981 \mu_{N}$, i.e. value overestimated by $2.6 \%$.
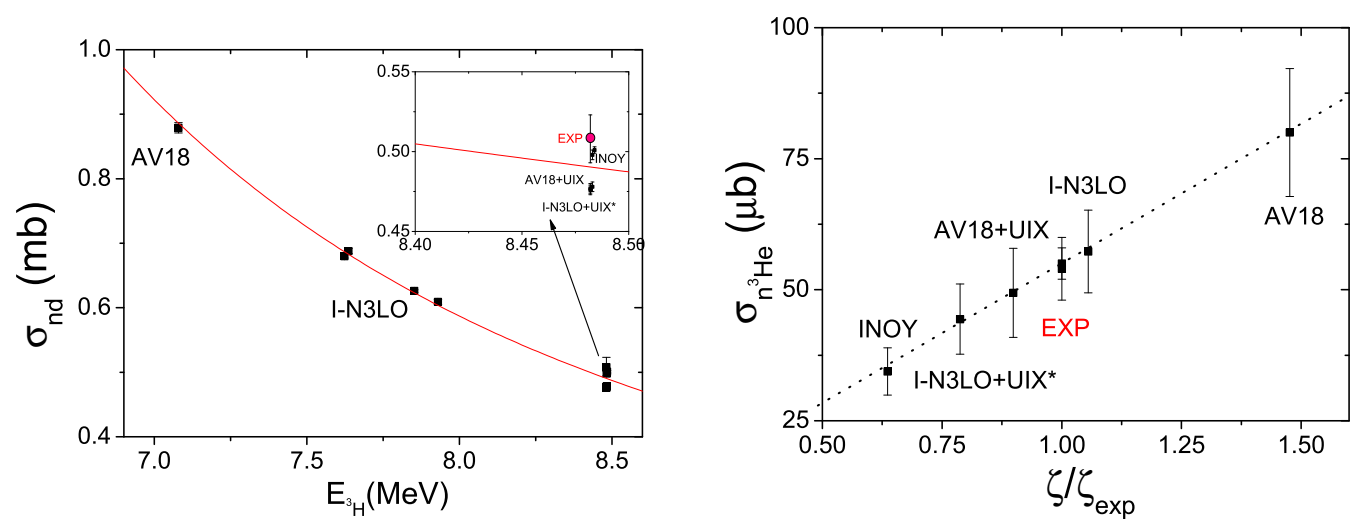

Figure 1: Correlation of the calculated den cross section with the ${ }^{3} \mathrm{H}$ binding energy (left pane). Correlation between the calculated hen cross section with $\zeta \equiv\left[q\left(a_{n \mathrm{He} 3} / r_{\mathrm{He} 4}\right)^{2}\right]^{-2.75}$ parameter (right pane).

Our hen results are the first ones obtained combining fully realistic nuclear wave functions and HBChPT currents. By correcting effects due to the effective range parameters controlling long range behavior of the systems wave function model independent prediction can be obtained, giving reasonable agreement with experimental data. Before the present work, the only ab initio calculation of the hen reaction has been performed by Schiavilla et al. in [27]. In last work the nuclear wave functions were obtained within a different approach (the variational Monte Carlo method), using the older Av14+UVIII Hamiltonian model and meson exchange current operators constructed in a Chemtob-Rho type of phenomenological way. Hen cross section calculated in [27] were $85.9 \mu \mathrm{b}$ and $112 \mu \mathrm{b}$, sizeably different from our results. This difference is not only due to the difference in MEC operators employed, since our calculated values already for one-body impulse approximation term are as much as 2.6 times larger than Schiavilla's. Part of this discrepancy can be attributed to the difference in calculated $n-{ }^{3} \mathrm{He}$ scattering length and the fact that coupling of $n-{ }^{3} \mathrm{He}$ channel with $p-{ }^{3} \mathrm{H}$ one has been ignored in work by Schiavilla et al. However the main reason can be due to the fact that the last variational calculations involved very few twobody correlation operators, which could be not enough to estimate impulse approximation operator coupling only the minor components of the initial and final nuclear states.

\section{Acknowledgement}

This work was granted access to the HPC resources of IDRIS under the allocation 2009i2009056006 made by GENCI (Grand Equipement National de Calcul Intensif). We thank the staff members of the IDRIS for their constant help. 


\section{References}

[1] T.-S. Park, D.-P. Min and M. Rho, Phys. Rev. Lett. 74 (1995) 4153; Nucl. Phys. A596 (1996) 515; T.-S. Park, K. Kubodera, D.-P. Min and M. Rho, Phys. Rev. C58 (1998) R637.

[2] T.-S. Park, K. Kubodera, D.-P. Min and M. Rho, Phys. Lett. B472 (2000) 232.

[3] C.H. Hyun, T.-S. Park and D.-P. Min, Phys. Lett. B516 (2001) 321.

[4] J.-W. Chen and M.J. Savage, Phys. Rev. C60 (1999) 065205; J.-W. Chen, G. Rupak and M.J. Savage, Phys. Lett. B464 (1999) 1.

[5] H. Sadeghi and S. Bayegan, Nucl. Phys. $\mathbf{A 5 3}$ (2005) 291; H. Sadeghi, S. Bayegan and H.W. Griesshammer, Phys. Lett. B643 (2006) 263; nucl-th/0610029; H. Sadeghi, Phys. Rev. C75 (2007) 044002 .

[6] R. Skibinski, J. Golak, H. Witala, W. Gloeckle, A. Nogga and E. Epelbaum, Acta Phys. Polon. B37 (2006) 2905.

[7] Y.-H. Song, R. Lazauskas and T.-S. Park, Phys. Lett. B656 (2007) 174.

[8] J. D. Walecka, Theoretical Nuclear and Subnuclear Physics (Oxford University Press, New York, 1995)

[9] E. Epelbaum, W. Gloeckle and U. G. Meissner, Nucl. Phys. A671, 295 (2000); E. Epelbaum, W. Gloeckle and U. G. Meissner, Nucl. Phys. A747, 362 (2005).

[10] Y.-H. Song, R. Lazauskas and T.-S. Park, Phys. Rev. C79, (2009) 064002.

[11] L. D. Faddeev: Zh. Eksp. Teor. Fiz. 39, (1960) (Wiley \& Sons Inc., 1972) 1459 [Sov. Phys. JETP 12, (1961) 1014].

[12] O. A. Yakubowsky, Sov. J. Nucl. Phys. 5 (1967) 937.

[13] R. Lazauskas, Ph.D. Thesis, Université Joseph Fourier, Grenoble (2003); http://tel.ccsd.cnrs.fr/documents/archives0/00/00/41/78/.

[14] R.B. Wiringa, V.G.J. Stoks, R. Schiavilla, Phys. Rev. C 51 (1995) 38.

[15] D.R. Entem and R. Machleidt, Phys. Rev. C 68 (2003) 041001(R).

[16] P. Doleschall, I. Borbély, Z. Papp, W. Plessas, Phys. Rev. C 67 (2003) 064005.

[17] B.S. Pudliner, V.R. Pandharipande, J. Carlson and R.B. Wiringa, Phys. Rev. Lett. 74 (1995) 4396.

[18] R. Lazauskas, Y.-H. Song and T.-S. Park, arXiv:0905.3119.

[19] S. F. Mughabghab, M. Divadeenam, and N. E. Holden, Neutron Cross Sections from Neutron Resonance Parameters and Thermal Cross Sections (Academic Press, London, 1981), http://isotopes.lbl.gov/ngdata/sig.htm.

[20] E.T. Jurney, P.J. Bendt, and J.C. Browne, Phys. Rev. C25 (1982) 2810.

[21] M.W. Konijnenberg, K. Abrahams, J. Kopecky, F. Stecher-Rassmussen, R. Wervelman, and J.H. Koch, Phys. Lett. B205 (1988) 215.

[22] F.L.H. Wolfs, S.J. Freedman, J.E. Nelson, M.S. Dewey and G.L. Greene, Phys. Rev. Lett. 63 (1989) 2721.

[23] R. Wervelman, K. Abrahams, H. Postma, J.G.L. Booten and A.G.M. Van Hees, Nucl. Phys. A526 (1991) 265 . 
[24] J.L. Friar, B.F. Gibson and G.L. Payne, Phys. Lett. B251 (1990) 11.

[25] M. Viviani, R. Schiavilla and A. Kievsky, Phys. Rev. C54 (1996) 534.

[26] S. Pastore, R. Schiavilla and J. L. Goity, arXiv:0809.2555 [nucl-th].

[27] R. Schiavilla, R. B. Wiringa, V. R. Pandharipande and J. Carlson, Phys. Rev. C 45 (1992) 2628.

J. Carlson, D. O. Riska, R. Schiavilla and R. B. Wiringa, Phys. Rev. C 42 (1990) 830. 